\title{
The Stokes Laser in the Different Pump Regimes
}

\author{
Dinh Xuan Khoa ${ }^{1}$, Chu Van Lanh ${ }^{1}$, Doan Hoai Son ${ }^{1}$, Ho Quang Quy ${ }^{2}$ \\ ${ }^{1}$ Faculty of Physics, Vinh University, Vietnam \\ ${ }^{2}$ Institute of Applied Physics, NEWTECHPRO, VAST, 8 Hoang Quoc Viet, Hanoi, Vietnam \\ hoquy1253@yahoo.com
}

(Received: 29 June 2010; accepted: 13 September 2010)

Abstract: In is paper we present the set of dynamical equations of the Raman laser. The semi-classical theory in three-dimensional approach is of interest. The operating regime of the Stokes laser is investigated in some cases of the pumping rate.

Key words: semi-classical theory, Stokes laser, pumping regime

\section{INTRODUCTION}

The Raman laser is a recently developed laser technique for generating tunable high-quality laser beam in the nearinfrared region. It is based on stimulated antiStokes scattering and Stokes scattering as illustrated in Fig. 1.

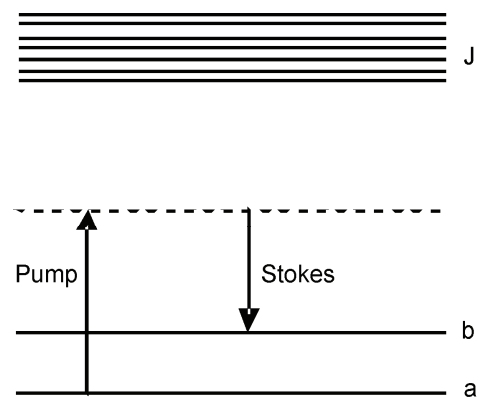

Fig. 1. Energy level diagram of the far-off-resonance Stokes Raman process

Level $a$ is the ground state, level $b$ is the molecular vibrational or rotational state, and levels $J$ are the excited electronic states far-off-resonant. The transition between levels $a$ and $b$ is electric dipole forbidden. Raman scattering occurs when an incident photon (called the pump) interacts with the molecule and generates a red-shift photon (called the Stokes) and a blue-shift photon (called the antistokes). Theory and experiment of Raman scattering and Raman laser (Fig. 2) are investigated in many works $[2,3,8]$. Up to now, the results of Raman laser theory have been limited in one-dimensional approach only. In the work [1], Raymer et al have discussed the stimulated Raman scattering in the three-dimensional approach.

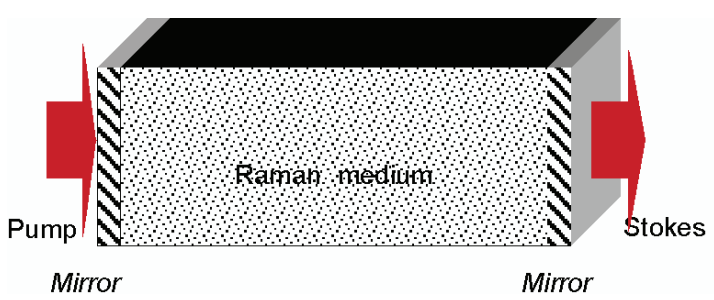

Fig. 2. Structure of Raman laser

In this paper, we develop a semi-classical theory for the far-off-resonance Raman laser in three-dimensional approach. The time-dependent solution to the continuouswave Stokes laser and pulse Stokes laser is numerically solved using the intraccavity field equations.

\section{FIELD AMPLITUDE EQUATION OF THE STOKES LASER}

We assume that the optical field inside the cavity will be in the single spatial mode and can be written as:

$$
E_{q}(\vec{r}, t)=E_{q}(t) u_{q}(\vec{r})
$$


where subscript $q$ will denote the various frequency components inside the cavity (length $L$ ) such as the pump and the Stokes. Every mode is orthogonal:

$$
\iiint_{\text {cavity }} u_{q}(\vec{r}) u_{q}^{*}(\vec{r}) d x d y d z=V_{q}
$$

where $V_{q}$ is the mode volume occupied by the $q$-th spatial mode.

Using the Maxwell's wave equation with slowly-varying envelope approximation, the density matrix equation with adiabatic elimination (for all time the upper levels $J$ are in steady state), we have the intracavity field equations [7]

$$
\begin{gathered}
\left|\dot{E}_{p}(r)\right|+\frac{\gamma_{p}}{2}\left|E_{p}(t)\right|(t)= \\
=-\frac{\omega_{p}}{\omega_{s}} \frac{k_{p}}{k_{s}} G(\delta)\left|E_{s}(t)\right|^{2}\left|E_{p}(t)\right|(t)+\frac{\gamma_{e p}}{2}\left|E_{p}(t)\right| \\
\left|\dot{E}_{s}(t)\right|+\frac{\gamma_{s}}{2}\left|E_{s}(t)\right|=G(\delta)\left|E_{p}(t)\right|^{2}\left|E_{s}(t)\right|
\end{gathered}
$$

where

$$
\begin{gathered}
\gamma_{e p}=\frac{2 c}{n_{p} L} \sqrt{T_{1 p}}, \\
\gamma_{(p) s}=-\frac{c}{n_{(p) s} L} \ln \sqrt{R_{1(p) s} R_{2(p) s}}
\end{gathered}
$$

present the intracavity energy decay rate primarily due to the loss of the mirrors (transmitance $T$, reflectance $R$ ), relating to the external pump field, intracavity pump field and Stokes field, respectively [3]. The gain term is defined as follows

$$
G(\delta)=\frac{\Phi^{2}}{8} c^{2} \varepsilon_{0} \alpha_{g}(\delta) \frac{\lambda_{p}}{\lambda_{p}+\lambda_{s}} \frac{\tan ^{-1}\left(L / b_{q}\right)}{L / b_{q}}
$$

with

$$
\begin{aligned}
& \alpha_{g}(\delta)=\alpha_{g}(0)\left(1+\frac{4\left|\Omega_{a b}\right|^{2}}{\gamma_{a b} \Gamma_{a b}}\right)^{-1} \times \\
& \times\left(1+\frac{\delta^{2}}{\gamma_{a b}^{2}} \frac{1}{1+4\left|\Omega_{a b}\right|^{2} / \gamma_{a b} \Gamma_{a b}}\right)^{-1}
\end{aligned}
$$

which is the plane-wave gain coefficient. Its value at zero

$$
\alpha_{g}(0)=\frac{2 \omega_{s} N \hbar d_{s}^{2}\left(-D^{s}\right)}{n_{s} n_{p} c^{2} \varepsilon_{0}^{2} \gamma_{a b}}
$$

is the line center value of the unsaturated or small-signal plane-wave gain coefficient. $b_{q}$ denotes the confocal parameter relating to the mode volume:

$$
V_{p(s)}=\frac{\pi L b_{q}}{4 k_{p(s)}},
$$

$\Omega_{a b}$ is the two-photon Rabi frequency, $\gamma_{a b}$ is the coherence dephasing rate, $\Gamma_{a b}$ is the population decay rate and $\delta$ is the two-photon detuning for the $a \rightarrow b$ transition, $N$ is the molecule density, $d_{s}$ is the coupling constant to be real number. Finally $D$ is the population difference, given as

$$
D=\frac{\Gamma_{a b} D^{e q}\left(\gamma_{a b}^{2}+\delta^{2}\right)}{\Gamma_{a b}\left(\gamma_{a b}^{2}+\delta^{2}\right)+4\left|\Omega_{a b}\right|^{2} \gamma_{a b}}
$$

where $D^{e q}$ is the population difference in thermal equilibrium equal -1 , i.e. all the population in the ground state. In the three-dimensional approach, the Fresnel number $\Phi=A / \lambda_{s} L$ describes the diffraction in a pencil-shape medium of cross-section $A$ and length $l$ [4].

\section{A SET OF RATE EQUATIONS FOR INTRACAVITY POWERS}

It is necessary to write the intensity field equation in terms of measurable powers, so that one should compare the experimental data with theory. In a stable two-mirror laser cavity of $L$ and in the $\mathrm{TEM}_{00}$ spatial mode, $u_{q}(\vec{r})$ is of the form [10]

$$
u_{q}(\vec{r})=u_{q}(r, z)=\frac{1}{1+i 2 z / b_{q}} e^{-r^{2} k_{q} / b_{q}\left(1+i 2 z / b_{q}\right)} \sin \left(k_{q} z\right)(10)
$$

where $r^{2}=x^{2}+y^{2}$.

From (1) and (10) one can see that $E_{q}(t)$ represents the peak filed-amplitude of the intracavity standing-wave in space (i.e. at the center of the $\mathrm{TEM}_{00}$ transverse profile and at the antinodes of the standing-wave along the longitudinal direction). The intracavity light intensity is calculated by

$$
I_{q}=\frac{v_{q} \varepsilon_{q}}{2}\left|E_{q}(t) u_{q}(r)\right|^{2}
$$

where $v_{q}=c / n_{q}$ is the intracavity light speed and $\varepsilon_{q}=n_{q}^{2} \varepsilon_{0}$ is the dielectric permeability of the intracavity medium. The intracavity power along the longitudinal direction can be calculated

$$
\begin{gathered}
P_{q}(z, t)=\int_{0}^{\infty} r d r \int_{0}^{2 \pi} d \varphi I_{q}= \\
=\frac{\pi \varpi_{0 q}^{2} n_{q}}{4} \sqrt{\frac{\varepsilon_{0}}{\mu_{0}}}\left|E_{q}(t)\right|^{2} \frac{\sin ^{2}\left(k_{q} z\right)}{1+\left(2 z / b_{q}\right)^{2}}
\end{gathered}
$$


where $\varpi_{0 q}=\sqrt{b_{q} / k_{q}}$ is the radius at the beam waist. If the beam inside the cavity is collimated $\left(z<<b_{q}\right)$, the peak intracavity power along the longitudinal direction is given by

$$
P_{q}(t)=\frac{\pi \varpi_{0 q}^{2} n_{q}}{4} \sqrt{\frac{\varepsilon_{0}}{\mu_{0}}}\left|E_{q}(t)\right|^{2}=\frac{\pi b_{q}}{4 \omega_{q} \mu_{0}}\left|E_{q}(t)\right|^{2} .
$$

A standing wave consists of two counter-propagating equal-amplitude traveling waves and the peak-amplitude ratio between the standing and traveling waves is $2: 1$ [11]. Therefore, the optical power of the traveling-waves is equal to $(1 / 4) P_{q}(t)$.

Using the relation Eq. (13), one can convert Eq. (3) into

$$
\begin{gathered}
\dot{P}_{p}(t)+\gamma_{p} P_{p}(t)= \\
=-\frac{8 \omega_{p} \mu_{0}}{\pi b} \frac{k_{p}}{k_{s}} G(\delta) P_{s}(t) P_{p}(t)+\gamma_{e p} \sqrt{P_{p}(t) P_{e p}(t)} \\
\dot{P}_{s}(t)+\gamma_{s} P_{s}(t)=\frac{8 \omega_{p} \mu_{0}}{\pi b} G(\delta) P_{s}(t) P_{p}(t) .
\end{gathered}
$$

From the steady-state solution, one can find the threshold pump power given by

$$
P_{e p, t h}=\frac{\left(\ln \sqrt{R_{1 p} R_{2 p}}\right)^{2}}{4 T_{1 p}} \frac{\pi b \gamma_{s}}{8 \omega_{p} \mu_{0} G(\delta)} .
$$

Note that the $P_{p}(t)$ and $P_{s}(t)$ are the peak intracavity power along the longitudinal direction.

Consider a pump pulse, which is a Gaussian function of time as follows:

$$
P_{e p}(t)=P_{\max } \exp \left(-\left(\frac{\sqrt{2} t}{\tau}\right)^{2}\right)
$$

Substituting into (14), one has a set of equations, describing the unstationary operation of the Stokes laser.

\section{INFLUENCE OF PUMP RATE}

For the time-dependent solution to the pulsed Stokes laser, we choose to numerically solve the intracavity Eqs. (14). For example, we numerically solve Eqs. (14) for the vibrational transition of $792 \rightarrow 1180 \mathrm{~nm} \quad\left(\omega_{p}=2 \pi c / 792 \times 10^{-9}\right.$, $\omega_{s}=2 \pi c / 1180 \times 10^{-9}$ ) for $\mathrm{H}_{2}$ at $25^{\circ} \mathrm{C}$ and $10 \mathrm{~atm}$ [5-7]). Therefore, the gain coefficient $\alpha_{g}=0.5 \times 10^{-7} \mathrm{~m} / \mathrm{W}$, the coherence decay for vibrational $\gamma_{a b} / 2 \pi=250 \mathrm{MHz}$, the two-photon detuning $\delta / 2 \pi \approx \pm 0.5 \mathrm{GHz}$, the molecular density $N=2.4 \times 10^{26} \mathrm{~m}^{-3}$, the coupling constant $d_{s} \approx 6.0 \times 10^{-8} \mathrm{~m}^{2} \mathrm{~Hz} / \mathrm{V}^{2}, \quad n_{s} \approx n_{p} \approx 1, D^{e q} \approx 1$, the popu- lation decay $\Gamma_{a b} / 2 \pi \approx 10 \mathrm{kHz}$, are chosen for molecules of the Raman medium. The reflectance of mirror $R_{1(2) s}=0.9, R_{1 p}=0.9, R_{2 p}=0.9$, the length $L=0.1 \mathrm{~m}$, the curvature radius $r=0.5 \mathrm{~m}, b_{s}=\sqrt{L(2 r-L)} \approx 0.3 \mathrm{~m}$, and the cross section of medium $A=12.3 \times 10^{-4} \mathrm{~m}^{2}$ and $l=0.04 \mathrm{~m}$ are chosen for the laser cavity. The peak power of external pump pulse is chosen to be 2,4 and $8, \ldots$ times the threshold, which is calculated from (15).

The time-dependent results for $\mathrm{CW}$ regime are given in Fig. 3 for the extracavity pump power and Fig. 4 for the intracavity Stokes one. It can be seen that at low pumping rate less than two times the threshold, the laser turns on smoothly and slowly, whereas at more than four times the threshold, there is a large overshoot. Moreover, the pump field and Stokes field pulse before reaching its stable magnitude, and the pulsing time decreases when the pump power increases. An interesting point is that the stable magnitude of intracavity pump power is constant, meanwhile the stable magnitude of Stokes power increases when the extracavity pump power increases.

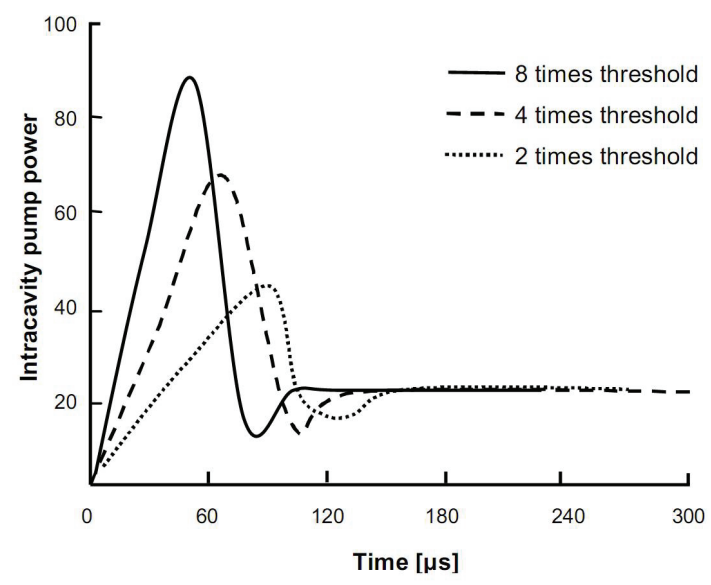

Fig. 3. The intracavity pump power $v$ s time

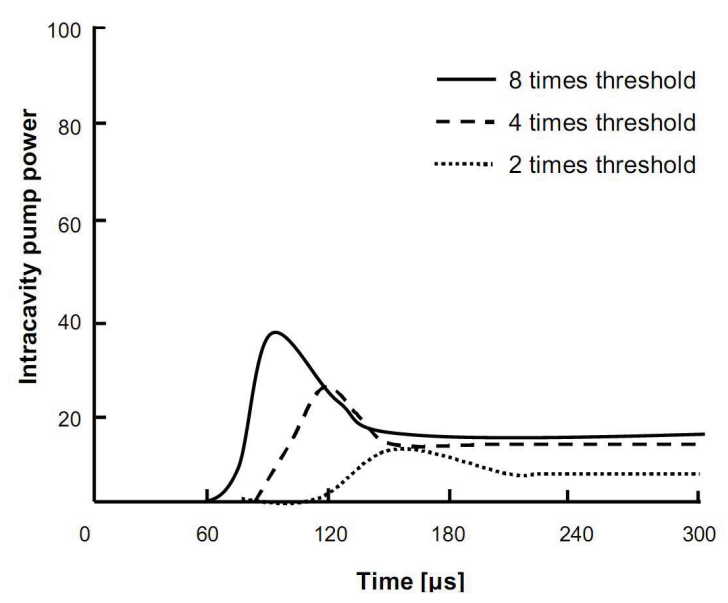

Fig. 4. The intracavity Stokes power $v$ s time 


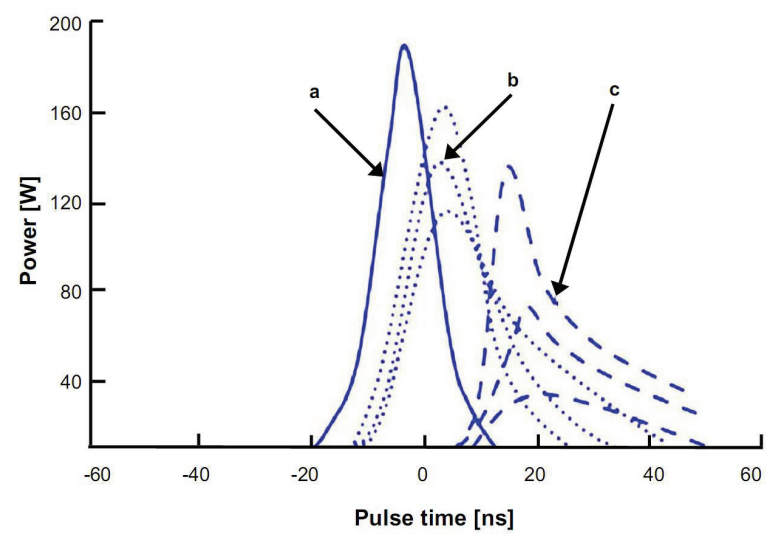

Fig. 5. Pulsing of the intracavity power and the intracavity Stokes power

For the unstationary operation, the pump pulse is chosen of $P_{\max }$ to be 1-3 $P_{e p, t h}$ and $\tau=10 \mathrm{~ns}$, for example. The intracavity power of the pump pulse and Stokes pulse are calculated numerically using Eq. (14) and (16), and presented in Fig. 5. From this figure one can see that the intracavity pump pulse and the intracavity Stokes pulse are delayed about $5 \mathrm{~ns}$ and $30 \mathrm{~ns}$ from pump pulse, respectively. The intracavity Stokes power is increasing strongly, when the peak of pump power increases. Moreover, the transfer power efficiency from pump field to Stokes field increases with increasing of $P_{\max }$.

\section{CONCLUSION}

The semi-classical theory of the Stokes laser in the three-dimention approach is derived. The intracavity power equation are introduced. The time-dependence of intracavity powers of the $\mathrm{CW}$ and pulse laser is numerically calculated. The calculated results for three cases of the extracavity power show that the intracavity power of Stokes field pulse before reaching a stable magnitude, which increases with the increasing of the intracavity power for $\mathrm{CW}$ regime, and Stokes pulse delays with a time interval from pump pulse and its peak power increases with increasing of extracavity pump one. Our results are important for investigation of the properties of the Stokes and its designing in future.

\section{References}

[1] M.G. Raymer et al., Phys. Rev. A 24, 1980 (1981).

[2] M.G. Raymer, L.A. Westling, J. Opt. Soc. Am.B, 2, 1417 (1985).

[3] R.W. Boyd, Nonlinear Optics, Academic Press (1992).

[4] P.A. Roos et al., J. Opt. Soc. B, 17, 758 (2000).

[5] J.K. Brasseur et al., J. Opt. Soc. Am. B, 17, 1229 (2000).

[6] F.L. Kien et al., Phys. Rev. A 60, 1562 (1999).

[7] L.S. Meng et al., Opt. Lett. 25, 472 (2000).

[8] J.K. Brasseur et al., Opt. Lett. 27, 390 (2002).

[9] R. Calps et al., Opt. Express 13, 2459 (2005).

[10] G.D. Boyd et al., IEEE J. Quantum Electron. 5, 203 (1969).

[11] J.K. Brasseur et al., J. Opt. Soc. Am. B 16, 1229 (1999).

[12] T.B. Chu, H. Q. Quy, Comm. in Phys. 8, 118 (1998).

[13] L.V. Taracov, Nonlinear Optics, "Mir", Moscov (1982).

[14] C.T. Le, H.Q. Quy, Comm. In Phys. 11, 76 (2001).
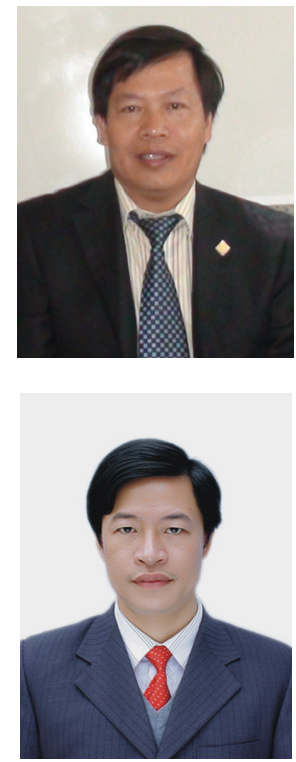

Dinh Xuan KhoA was born in Nghe An province, Vietnam. He graduated from Vinh University in 1981. He completed a PhD course in Quantum Optics in 1996. His doctoral thesis entitled "Physics of Dye Lasers" was supervised by Prof. Cao Long Van and Prof. Dao Xuan Hoi. Since 1997, he has been head of the research group of Optics at Vinh University. He was elected as associate professor in Physics in 2003. His field of interests covers a large variety of topics in Quantum and Nonlinear Optics, Atomic and molecular Physics.

Chu Van Lanh received his master degree in Physics at the Vinh University in Vietnam in 2001 and Doctor degree in Physics at Vinh University in Vietnam in 2009. His current field of interest concerns quantum optics and laser physics. 


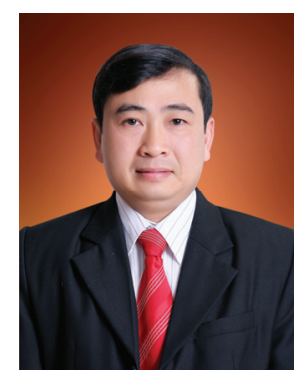

DoAN HoAI Son was born in Ha Tinh province, Vietnam. He graduated, with a major in Physics, from Vinh University in 1992. He completed a PhD course in Optics in 2006 under the supervison of professor Nguyen Dai Hung. Since 2008 he has been pointed as the dean of the Faculty of Physics. His field of interests covers a large variety of topics in Nonlinear Optics, Laser physics.

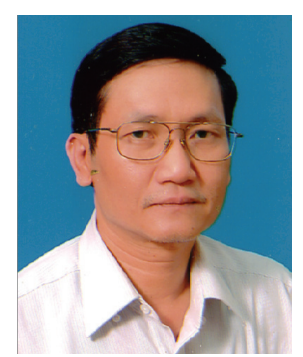

Ho QUang QuY received his M.Sc. degree in Physics in 1978 from M.Copernicus University in Torun, Poland; his Ph.D. degree in quantum-electronics from the Academy of Military Scientist and Technology in 1992, and Professor title from Education-Training Ministry of Viet Nam in 2002. Over nearly 30 years of his scientific life he has had more than 60 publications. Now, he is the researcher and expert in laser spectroscopy and nonlinear physics, and interested in optical trapping and optical bistability. 